\title{
Systemic inflammation: a key factor in the pathogenesis of cardiovascular complications in obstructive sleep apnoea syndrome?
}

\author{
S Ryan, ${ }^{1,2}$ C T Taylor, ${ }^{2}$ W T McNicholas ${ }^{1,2}$
}

\begin{abstract}
${ }^{1}$ Sleep Research Laboratory, St Vincent's University Hospital, Dublin, Ireland; ${ }^{2}$ School of Medicine and Medical Science, The Conway Institute, University College Dublin, Ireland
\end{abstract}

Correspondence to: Professor W T McNicholas, Department of Respiratory Medicine, St Vincent's University Hospital, Elm Park, Dublin 4, Ireland: walter.mcnicholas@ucd.ie

Received 29 July 2008 Accepted 6 November 2008

\section{ABSTRACT}

Obstructive sleep apnoea syndrome (OSAS) is a highly prevalent disease and is recognised as a major public health burden. Large-scale epidemiological studies have demonstrated an independent relationship between OSAS and various cardiovascular disorders. The pathogenesis of cardiovascular complications in OSAS is not completely understood but a multifactorial aetiology is likely. Inflammatory processes have emerged as critical in the pathogenesis of atherosclerosis at all stages of atheroma formation. Increased levels of various circulating markers of inflammation including tumour necrosis factor $\alpha$ (TNFa), interleukin 6 (IL6), IL-8 and C-reactive protein (CRP) have been reported as associated with future cardiovascular risk. There is increasing evidence of elevated inflammatory markers in OSAS with a significant fall after effective treatment with continuous positive airway pressure. This evidence is particularly strong for TNF $\alpha$, whereas studies on IL6 and CRP have yielded conflicting results possibly due to the confounding effects of obesity. Cell culture and animal studies have significantly contributed to our understanding of the underlying mechanisms of the association between OSAS and inflammation. Intermittent hypoxia, the hallmark of OSAS, results in activation of pro-inflammatory transcription factors such as nuclear factor kappa B (NF-кB) and activator protein (AP)-1. These promote activation of various inflammatory cells, particularly lymphocytes and monocytes, with the downstream consequence of expression of pro-inflammatory mediators that may lead to endothelial dysfunction. This review provides a critical analysis of the current evidence for an association between OSAS, inflammation and cardiovascular disease, discusses basic mechanisms that may be responsible for this association and proposes future research possibilities.

Obstructive sleep apnoea syndrome (OSAS) is characterised by instability of the upper airway during sleep resulting in markedly reduced (hypopnoea) or absent (apnoea) airflow at the nose/ mouth. These episodes are usually accompanied by loud snoring and oxyhaemoglobin desaturation, and are typically terminated by brief arousals which result in marked sleep fragmentation and diminished amounts of slow wave and rapid eye movement (REM) sleep. ${ }^{1}$ Patients with OSAS are usually unaware of this sleep disruption, but the changes in sleep architecture contribute significantly to the prominent symptom of chronic daytime sleepiness found in these patients. The prevalence of OSAS among the adult population is high, being $4 \%$ in men and $2 \%$ of women in the Wisconsin Sleep Cohort Study. ${ }^{2}$ Given the rapidly rising incidence of obesity, which is the most important risk factor of OSAS, the prevalence is now likely to be significantly higher. ${ }^{3}$

The current management of moderate to severe OSAS is largely dependent on nasal continuous positive airway pressure (CPAP), which acts to splint the upper airway open during sleep and thus counteracts the negative suction pressure during inspiration that promotes upper airway collapse in these patients. ${ }^{45}$ Nasal CPAP completely controls the condition and has a dramatic effect on the patient's awake performance because of the normalised sleep pattern.

OSAS is associated with significant morbidity and mortality. The excessive daytime sleepiness leads to impairments in quality of life, cognitive performance and social functioning. ${ }^{6}$ Furthermore, the disorder is associated with a $3-7$-fold increase in the rate of road traffic accidents. ${ }^{7}$ The major health burden in patients with OSAS, however, is the strong risk of cardiovascular diseases such as systemic arterial hypertension, coronary artery disease, heart failure and stroke. ${ }^{8}$ The association between OSAS and cardiovascular diseases has been suggested for many years and, more recently, is corroborated by large-scale epidemiological and prospective studies. The underlying mechanisms mediating this association are incompletely understood. Current evidence suggests that inflammatory processes leading to endothelial dysfunction play a pivotal role in the pathogenesis.

Following a brief summary of the current epidemiological and clinical evidence of OSASassociated cardiovascular involvement and an overview of inflammatory processes in the atherosclerotic process, this article provides a critical review of the current evidence of an association between OSAS and systemic inflammation, describes basic mechanisms that may be responsible for this association and outlines future research perspectives.

\section{OSAS AND CARDIOVASCULAR DISEASES}

An association between OSAS and the development of cardiovascular diseases has been suggested for several years. However, confounding variables such as obesity, hypertension, smoking, alcohol intake, age and level of exercise have made this independent relationship difficult to prove. Furthermore, many of the earlier studies on OSAS and cardiovascular diseases used samples from clinical populations which are usually not representative of the condition in the general population, and cannot be used to estimate the 
public health impact of the disease. ${ }^{3}$ However, the evidence of an association is growing, particularly with systemic arterial hypertension. Both the Sleep Heart Health Study which included over 6000 subjects and the Wisconsin Sleep prospective cohort study involving 1069 subjects have yielded convincing evidence of a modest but definite association, independent of possible confounding factors such as age, sex and obesity. ${ }^{9} 10$ The prevalence of OSAS is particularly high in patients with drug-resistant hypertension; a recent study found occult OSAS in up to $83 \%$ of patients who had uncontrolled hypertension despite taking three or more antihypertensive agents at optimum doses. ${ }^{11}$

Data linking OSAS to other cardiovascular diseases are not as clear-cut but, nonetheless, supportive. The strongest evidence is provided by data from the Sleep Heart Health Study cohort which reports an independent association between OSAS and congestive cardiac failure, cerebrovascular disease and coronary artery disease. ${ }^{12}$ In support, Peker et al followed patients with known coronary artery disease for 5 years and observed a significantly higher mortality in patients with an apnoea/ hypopnoea index (AHI) of $\geqslant 10 / \mathrm{h}$ in comparison with matched controls. $^{13}$

A key question relates to the effect of CPAP therapy for OSAS on cardiovascular outcomes. Clearly, the ultimate answer to this question is still outstanding due to the lack of a long-term randomised trial. However, the withholding of effective CPAP therapy from patients with severe OSAS over a long period of time is unethical because of the recognised benefit of CPAP to daytime sleepiness and related symptoms in addition to the reduction in the risk of driving accidents. Three long-term cardiovascular outcome studies have compared patients on effective CPAP therapy with untreated or incompletely treated patients over 7-10 years and consistently found a significantly increased mortality and morbidity in the untreated group. ${ }^{14-16}$ However, patients intolerant of CPAP or unwilling to use the device do not represent a desirable control group as they may not be compliant with other medications. Randomised controlled studies looking at the effect of CPAP therapy on $24 \mathrm{~h}$ blood pressure in general have yielded modest but significant reductions in blood pressure, both during the night and day, ${ }^{17}{ }^{18}$ and the beneficial effects were greatest in patients with preexisting hypertension. Furthermore, there is now good evidence suggesting a beneficial effect of CPAP on left ventricular function. Two recent randomised trials have shown significant improvements in left ventricular ejection fraction following

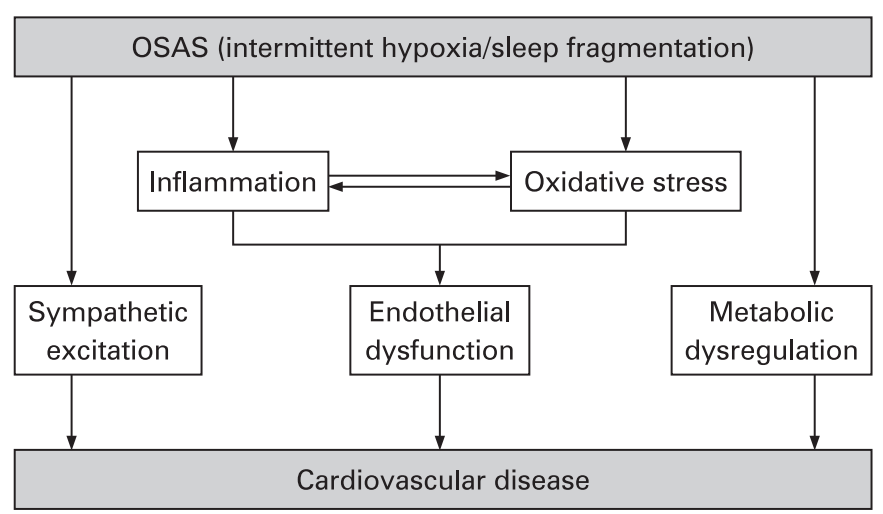

Figure 1 Mechanisms associated with obstructive sleep apnoea syndrome (OSAS) contributing to cardiovascular diseases.
CPAP therapy for 1-3 months in patients with systolic congestive cardiac failure and OSAS. ${ }^{19} 20$

In summary, there is growing evidence of a causal link between OSAS and the development of cardiovascular diseases, and CPAP therapy potentially decreases cardiovascular morbidity and mortality. Given the high and steadily rising prevalence of OSAS, the clarification of its exact role as a cardiovascular risk factor is a major priority. Identification of the detailed mechanisms underlying cardiovascular disease in patients with OSAS will help to support the current epidemiological evidence.

These mechanisms are, so far, still poorly understood. The pathogenesis is likely to be a multifactorial process involving a diverse range of mechanisms including sympathetic excitation, endothelial dysfunction and metabolic dysregulation (fig 1). ${ }^{8}$ Current evidence suggests that inflammatory processes leading to endothelial dysfunction play a pivotal role in the pathogenesis. Endothelial dysfunction substantially contributes to the development of various cardiovascular disease processes, particularly atherosclerosis but also hypertension and congestive cardiac failure.

\section{INFLAMMATION AND ATHEROSCLEROSIS}

Over the last several years we have seen major developments in our understanding of the pathogenesis of atherosclerosis. Inflammatory mechanisms have emerged as playing a pivotal role in all stages of atherosclerotic plaque formation, from initiation of the fatty streak to the culmination in plaque rupture presenting as acute coronary syndrome. ${ }^{21} 22$ Systemic inflammation occurs in the vasculature as a response to injury, lipid peroxidation and perhaps infection. ${ }^{23}{ }^{24}$ Resident or circulating leucocytes mediate the adherence of monocytes to the endothelium which in turn release a number of inflammatory mediators including cytokines such as tumour necrosis factor $\alpha(T N F \alpha)$ or interleukin (IL)-1, chemokines such as IL-8 or monocyte chemoattractant protein-1 (MCP-1) and adhesion molecules such as intercellular adhesion molecule 1 (ICAM-1) or selectins. Expression of adhesion molecules and chemokines facilitates the recruitment of macrophages, differentiated from monocytes, laden with oxidised lipid (foam cells). The accumulation of foam cells leads to the formation of a lipid pool, and collagen produced by smooth cells contributes to the strength of the fibrous cap. In particular, smooth cells also release IL6 which is the main hepatic stimulus for the acute phase reactant, C-reactive protein (CRP), which causes expression of adhesion molecules and mediates MCP-1 induction. ${ }^{25}$

\section{EVIDENCE OF INFLAMIMATION IN OSAS}

\section{Circulating inflammatory markers in OSAS}

The importance of inflammatory processes in the pathogenesis of cardiovascular diseases in OSAS is strongly supported by numerous studies demonstrating raised levels of circulating proinflammatory cytokines, chemokines and adhesion molecules in patients with OSAS in comparison with matched controls, and a significant fall with effective CPAP therapy. In particular, the potent pro-inflammatory cytokine TNF $\alpha$ has been evaluated by several case-control studies that have consistently shown raised levels in patients with OSAS compared with controls, independent of obesity, and a significant fall with effective CPAP therapy; both $\mathrm{T}$ cells and monocytes have been suggested as potential sources. ${ }^{26-30}$ Recently, a large prospective study in men without cardiovascular diseases identified a strong association between OSAS severity and TNF $\alpha$ levels, independent of 
possible confounders such as body mass index (BMI), age or sleepiness. ${ }^{29}$ Levels of the chemokine IL-8, which plays a key role in the process of adhesion of neutrophils and monocytes to the vascular endothelium, ${ }^{31}{ }^{32}$ have also been shown to be raised in OSAS. ${ }^{29}{ }^{33}$ Earlier studies have suggested increased IL6 levels in patients with OSAS, ${ }^{26} 3435$ but some of these reports may have been limited by smaller numbers, lack of adequately matched normal control populations, particularly in terms of BMI, and the inclusion of patients with established cardiovascular or metabolic diseases. Recent studies did not detect an association between OSAS and IL6. ${ }^{29}{ }^{36}$ However, in a large cross-sectional analysis of the Cleveland Family Study there was an independent association between OSAS severity parameters and soluble IL6 receptor, ${ }^{36}$ which appears to be associated with the processes of inflammation and myocardial injury during the acute phase of acute myocardial infarction. ${ }^{37}$

A limited number of studies have also examined the levels of various cellular adhesion molecules such as intercellular adhesion molecule 1 (ICAM-1), vascular adhesion molecule 1 (VCAM-1) and the family of selectins. The results consistently suggest an association between OSAS severity and circulating levels of cellular adhesion molecules, with one report also showing a significant fall after 1 month of effective CPAP therapy. ${ }^{38-42}$

Another potential link between OSAS and inflammation is the acute phase reactant C-reactive protein (CRP). In the high to normal range, when measured with a high-sensitivity assay, CRP levels are widely recognised as potent predictors of future cardiovascular events in apparently healthy subjects ${ }^{43} 44$ as well as in subjects with known cardiovascular disease. ${ }^{45}{ }^{46}$ However, recent large-scale studies suggest that the elevated levels may, in fact, be attributable to the presence of abnormal conventional cardiovascular risk factors, particularly obesity. ${ }^{47-49}$ The strong relationship between CRP levels and obesity has also influenced various studies investigating CRP levels in adults with OSAS, and therefore the role of CRP in OSAS is still under debate. This is reflected, for instance, in different conclusions obtained from two large cross-sectional studies in patients with OSAS. A study on 316 Japanese men detected a significant association between CRP and sleep-disordered breathing; however, the use of overnight oximetry as a screening tool for OSAS was a significant limitation. ${ }^{50}$ On the other hand, the Wisconsin Sleep Cohort Study involving 907 adults failed to detect an independent association between CRP and OSAS after adjustment for BMI. ${ }^{1}$ This discrepancy has also been evident in numerous case-control studies where some reports have identified increased levels of CRP in patients with OSAS ${ }^{34}$ 52-54 and others did not. ${ }^{5-57}$ Furthermore, the impact of CPAP therapy on CRP levels is still unclear. $^{34}$ 57-59 In a recent randomised trial comparing therapeutic and subtherapeutic CPAP, effective OSAS treatment had no significant effect on levels of CRP or IL6. ${ }^{60}$

It is noteworthy that the role of increased inflammatory markers in the prediction of cardiovascular morbidity in OSAS is still unclear and long-term prospective studies on this subject are lacking. Furthermore, vascular inflammation leading to endothelial dysfunction may potentially occur and persist in the absence of a systemic inflammatory response. A recent study provides evidence of increased expression of the inflammatory markers inducible nitric oxide synthase (iNOS) and cyclooxygenase-2 (COX-2) in harvested venous endothelial cells of patients with OSAS compared with matched controls, together with evidence of altered endothelial repair capacity that was reversed by CPAP therapy. ${ }^{61}$ However, whether these alterations are associated with a detectable systemic inflammation is unknown.

\section{Inflammatory cells and interactions with the endothelium in OSAS}

Accumulation of leucocytes and their adhesion to the endothelium play a central role in the formation of atherosclerotic plaques. Activation of monocytes and $\mathrm{T}$ lymphocytes is among the crucial steps leading to the release of inflammatory mediators and adhesion molecules.

Activation of inflammatory cells and their interaction with endothelial cells have been demonstrated in OSAS. In a rat model, recurrent obstructive apnoeas led to a significant increase in various leucocyte-endothelial cell interactions such as leucocyte rolling and firm adhesion of leucocytes in comparison with a sham group. ${ }^{62}$ Monocytes of patients with OSAS adhere more firmly to endothelial cells than those of control subjects, a process that is decreased by the application of CPAP therapy. ${ }^{63}$ In the same study, OSAS was associated with the upregulation of the adhesion molecules CD15 and CD11c in monocytes. Furthermore, in a cell culture model of repetitive hypoxia and reoxygenation, lipid uptake into macrophages and the expression of various adhesion molecules were significantly increased in comparison with control cells. ${ }^{64}$ Further evidence is provided in the report by Minoguchi et al who demonstrated significantly higher spontaneous $\mathrm{TNF} \alpha$ production by monocytes in patients with OSAS than in matched controls. ${ }^{30}$

$\mathrm{T}$ lymphocytes are also involved in the pathogenesis of atherosclerosis. In a series of experiments, Dyugovskaya et al showed that various subpopulations of cytotoxic $T$ cells of patients with OSAS acquire an activated phenotype with the downstream consequence of increased cytotoxicity against endothelial cells. ${ }^{27}{ }^{65}{ }^{66}$ Furthermore, this activation process is associated with an increased intracellular content of the proinflammatory mediators TNF $\alpha$ and IL-8 and a decrease of the anti-inflammatory cytokine IL-10. ${ }^{27}$

A recent in vitro study addressed the involvement of neutrophils in the cardiovascular pathogenesis of OSAS. ${ }^{67}$ The results demonstrate impaired neutrophil apoptosis and increased adhesion molecule expression by these cells in OSAS, thus suggesting a further potential pathway in the atherosclerotic process.

\section{MECHANISMS OF INFLAMMATORY PROCESSES IN OSAS}

The basic mechanisms underlying the inflammatory process in OSAS remain unclear. In addition to sleep fragmentation and sleep deprivation, the unique form of hypoxia in OSAS - with repetitive short cycles of desaturation followed by rapid reoxygenation, termed intermittent hypoxia $(\mathrm{IH})$ - is likely to play a significant role in the initiation of the inflammatory process. Various effects of IH on the cardiovascular system have been demonstrated using animal models. Rat and dog models of $\mathrm{IH}$ have consistently reported an increase in arterial blood pressure which is sustained even after cessation of the stimulus. ${ }^{68} 69$ Furthermore, enhanced sympathetic activity and decreased baroreflex sensitivity have been proposed to lead to the blood pressure increase in these models. ${ }^{70-72}$

There are various animal models supporting the development of atherosclerosis in response to $\mathrm{IH}^{73}{ }^{74}$ In mice on a highcholesterol diet, IH led to the development of atherosclerotic lesions which was not observed in control animals that were not exposed to $\mathrm{IH}^{73}$ Furthermore, cardiovascular remodelling with altered adhesion molecule expression has been observed in 
mice exposed to 14 days of IH. Interestingly, the right heart was not affected in this model whereas both the left ventricle and aorta demonstrated these changes. ${ }^{74}$

We recently showed in a cell culture model of $\mathrm{IH}$ a preferential activation of inflammatory pathways mediated by the transcription factor nuclear factor kappa B (NF-кB) over adaptive hypoxia-inducible factor 1 (HIF-1)-dependent pathways, which contrasts with sustained hypoxia where activation of adaptive and protective pathways predominate. ${ }^{28} \mathrm{NF}-\kappa \mathrm{B}$ is a key player in inflammatory and innate immune responses and a master regulator of inflammatory gene expression, and genes such as TNF $\alpha$ or IL-8 that are important to the atherosclerotic process and which have also been found upregulated in OSAS are under the control of this transcription factor. The central role of NF- $\kappa \mathrm{B}$ in inflammatory processes in OSAS was furthermore suggested by increased activation in cardiovascular tissues in a mouse model of $\mathrm{IH}$ and also in cultured monocytes of patients with OSAS. ${ }^{75}$ The p38 mitogen-activated protein kinase (MAPK) plays a major role in the process of IH-induced NF- $\kappa B$ activation, and pharmacological as well as targeted siRNA inhibition of p38 leads to a significant reduction in NF$\kappa \mathrm{B}$ activity. ${ }^{77}$ p38 MAPK is a key player in inflammatory processes and is necessary for inflammatory cytokine production and signalling. ${ }^{78}$ Furthermore, p38 is activated in response to environmental stresses and is critically involved in the pathophysiology of a variety of cardiovascular diseases. ${ }^{79-84}$ Activation of p38 MAPK was also demonstrated in a rat model of IH which was associated with delayed cardioprotection against prolonged ischaemia. ${ }^{85}$

Although our cell culture model demonstrates preferential activation of inflammatory over adaptive pathways by $\mathrm{IH}^{28}$ more prolonged exposure to IH may activate HIF-1, and this finding has been reported in another cell culture mode ${ }^{86}$ and in a mouse model of $\mathrm{IH}^{87}$ In particular, Yuan and co-workers reported increased HIF-1 transcriptional activity in response to 60 and 120 cycles of $\mathrm{IH}$ but not with fewer cycles. ${ }^{86}$

$\mathrm{IH}$ also activates other inflammatory transcription factors. Among them is the activator protein complex-1 (AP-1), formed by the proteins c-Fos and c-Jun. AP-1 drives transcriptional activation of a variety of genes including tyrosine hydroxylase which encodes the key enzyme in catecholamine synthesis. ${ }^{88} \mathrm{c}$ Fos upregulation by IH has been demonstrated in an animal as well as in a cell culture model. ${ }^{86} 89$ In a rat model, activation of inflammatory pathways by IH was associated with an impairment of neurocognitive function, a process which was reversed once the stimulus was removed. ${ }^{90}$

Yet to be determined remain(s) the initial sensing and signalling event(s) which occur(s) in response to IH. It has been proposed that repetitive episodes of hypoxia-and particularly of reoxygenation-lead to an increased production of reactive oxygen species (ROS). ${ }^{91}$ In support of this proposal, increased circulating levels of markers of oxidative stress have been found in patients with OSAS, and in vitro studies have demonstrated increased ROS production from leucocytes of patients with OSAS which was reversed by CPAP therapy. ${ }^{63}$ However, the involvement of ROS in NF- $\mathrm{KB}$ signalling is controversial, and experiments by Hayakawa et al indicate that NF- $\mathrm{KB}$ is unlikely to be a sensor of oxidative stress and previous results may have been influenced by cell type dependency and methodological limitations. ${ }^{92}$ In support of this, we did not detect an influence of the ROS scavenger N-acetyl-L-cysteine on NF- $\kappa B$ activation by $\mathrm{IH}$ in our cell culture model (unpublished data).

Collectively, the available evidence indicates that activation

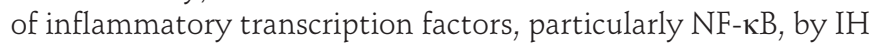

is critical in the pathogenesis of atherosclerosis in patients with OSAS.

\section{INTERACTION WITH OBESITY}

It is important to distinguish the inflammatory responses directly related to OSAS from obesity-related inflammation. Obesity, particularly visceral adiposity, is associated with chronic low-grade inflammation, as indicated by increased levels of the inflammatory markers CRP and IL6 in the circulation of obese subjects. ${ }^{93}$ Adipose tissue produces and releases a variety of pro-inflammatory and anti-inflammatory factors, including the adipokines leptin, adiponectin and resistin, as well as cytokines and chemokines such as IL6, $\mathrm{TNF} \alpha$, monocyte chemoattractant protein 1 (MCP-1) and others. These molecules produced by adipose tissue have been implicated as active participants in the development of insulin resistance and cardiovascular diseases. ${ }^{94}$ Thus, both obesity and OSAS are pro-inflammatory conditions and may mutually enhance the progression and severity of cardiovascular diseases.

\section{FUTURE RESEARCH PERSPECTIVE}

While substantial progress has been made in the identification of inflammatory processes in the cardiovascular pathogenesis of OSAS, a significant number of questions remain unanswered. The impact of CPAP therapy on cardiovascular diseases in OSAS has not been fully investigated, but may prove difficult to answer as a long-term randomised trial faces ethical problems. Understanding the basic molecular mechanisms of cardiovascular complications in OSAS can help to fill the gap of missing epidemiological evidence and, therefore, research in this field should be a major priority. Few translational studies have been undertaken so far that have explored basic mechanisms of

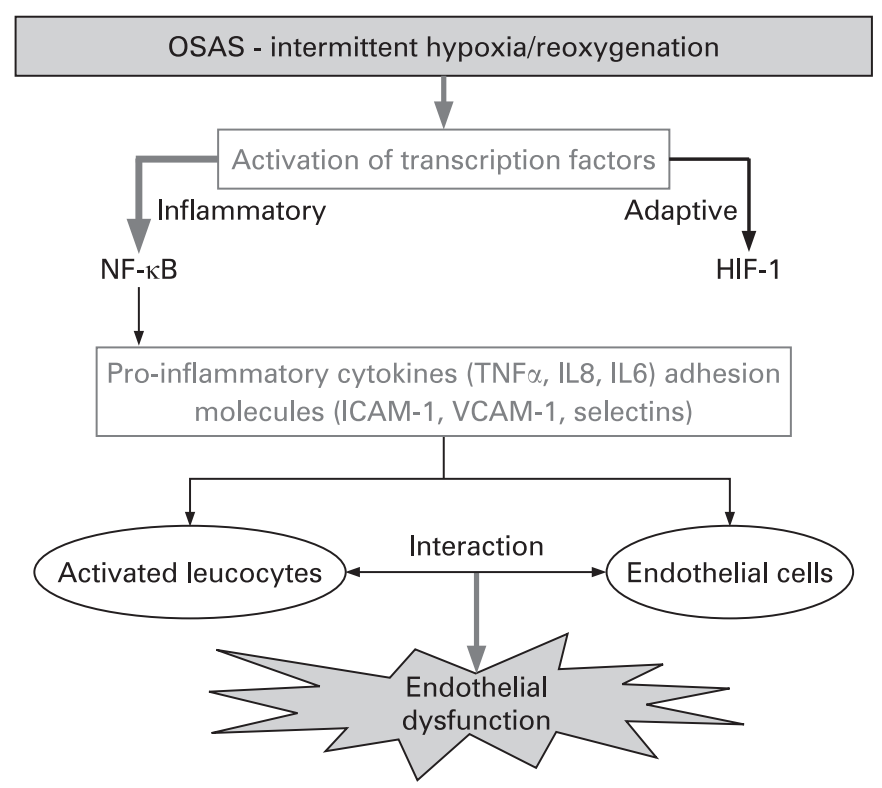

Figure 2 Selective activation of inflammatory pathways by intermittent hypoxia. Intermittent hypoxia leads to a preferential activation of nuclear factor kappa $B$ (NF- $\kappa B$ )-dependent inflammatory pathways over adaptive hypoxia-inducible factor 1 (HIF-1) mediated pathways. This results in the production of various pro-inflammatory mediators which, in turn, mediates the interaction of inflammatory and endothelial cells resulting in endothelial dysfunction. ICAM-1, intercellular adhesion molecule 1;

IL, interleukin; OSAS, obstructive sleep apnoea syndrome; TNF $\alpha$, tumour necrosis factor $\alpha$; VCAM-1, vascular adhesion molecule 1. 
cardiovascular diseases in OSAS with application of the findings to the clinical setting. Many of the clinical studies looking at inflammatory markers in OSAS have been influenced by small numbers, inadequately matched populations (particularly for BMI) and inclusion of patients with established cardiovascular or metabolic diseases. It is thus not surprising that conflicting results have been reported.

There is therefore a clear need for large-scale multicentre studies of carefully defined patient and control populations. Such studies carry the prospect of evaluating potential interactions between different basic mechanisms and cardiovascular disease and providing potential treatment options. As this review outlines, the basic inflammatory mechanisms underlying cardiovascular complications in OSAS are complex. There is emerging evidence that IH plays a central role in the initiation of the inflammatory processes, but additional studies involving cell, animal and human models are required to adequately explore the mechanisms involved.

\section{CONCLUSIONS}

Inflammatory processes are rapidly emerging as playing a central role in the cardiovascular pathogenesis of OSAS. The repetitive cycles of short periods of hypoxia followed by rapid reoxygenation preferentially activate inflammatory-particularly NF- $\kappa \mathrm{B}$-mediated-pathways. These mechanisms result in activation of inflammatory cells, release of inflammatory mediators and associated vascular pathophysiology (fig 2). Future therapeutic approaches to ameliorating the cardiovascular risk factors associated with OSAS should take into account the possibility of inhibiting systemic inflammatory processes.

Funding: Health Research Board (Ireland), Science Foundation of Ireland, Wellcome Trust

Competing interests: None.

\section{REFERENCES}

1. Guilleminault C, Tilkian A, Dement WC. The sleep apnea syndromes. Annu Rev Med 1976:27:465-84.

2. Young T, Palta M, Dempsey J, et al. The occurrence of sleep-disordered breathing among middle-aged adults. N Engl J Med 1993;328:1230-5.

3. Young T, Peppard PE, Gottlieb DJ. Epidemiology of obstructive sleep apnea: a population health perspective. Am J Respir Crit Care Med 2002;165:1217-39.

4. Deegan PC, McNicholas WT. Pathophysiology of obstructive sleep apnoea. Eur Respir J 1995;8:1161-78.

5. Sullivan CE, Issa FG, Berthon-Jones M, et al. Reversal of obstructive sleep apnoea by continuous positive airway pressure applied through the nares. Lancet 1981;1:862-5

6. Engleman HM, Douglas NJ. Sleep. 4: Sleepiness, cognitive function, and quality of life in obstructive sleep apnoea/hypopnoea syndrome. Thorax 2004;59:618-22.

7. Stoohs RA, Bingham LA, Itoi $A$, et al. Sleep and sleep-disordered breathing in commercial long-haul truck drivers. Chest 1995;107:1275-82.

8. McNicholas WT, Bonsigore MR. Sleep apnoea as an independent risk factor for cardiovascular disease: current evidence, basic mechanisms and research priorities. Eur Respir J 2007;29:156-78.

9. Nieto FJ, Young TB, Lind BK, et al. Association of sleep-disordered breathing, sleep apnea, and hypertension in a large community-based study. Sleep Heart Health Study. JAMA 2000;283:1829-36.

10. Peppard PE, Young T, Palta M, et al. Prospective study of the association between sleep-disordered breathing and hypertension. N Engl J Med 2000;342:1378-84.

11. Logan AG, Perlikowski SM, Mente A, et al. High prevalence of unrecognized sleep apnoea in drug-resistant hypertension. J Hypertens 2001;19:2271-7.

12. Shahar E, Whitney CW, Redline S, et al. Sleep-disordered breathing and cardiovascular disease: cross-sectional results of the Sleep Heart Health Study. Am J Respir Crit Care Med 2001;163:19-25.

13. Peker Y, Hedner J, Kraiczi H, et al. Respiratory disturbance index: an independent predictor of mortality in coronary artery disease. Am J Respir Crit Care Med 2000;162:81-6.

14. Peker Y, Hedner J, Norum J, et al. Increased incidence of cardiovascular disease in middle-aged men with obstructive sleep apnea: a 7-year follow-up. Am J Respir Crit Care Med 2002;166:159-65.
15. Marin JM, Carrizo SJ, Vicente E, et al. Long-term cardiovascular outcomes in men with obstructive sleep apnoea-hypopnoea with or without treatment with continuous positive airway pressure: an observational study. Lancet 2005;365:1046-53.

16. Doherty LS, Kiely JL, Swan V, et al. Long-term effects of nasal continuous positive airway pressure therapy on cardiovascular outcomes in sleep apnea syndrome. Chest 2005; 127:2076-84.

17. Becker HF, Jerrentrup A, Ploch $T$, et al. Effect of nasal continuous positive airway pressure treatment on blood pressure in patients with obstructive sleep apnea. Circulation 2003;107:68-73.

18. Pepperell JC, Ramdassingh-Dow S, Crosthwaite N, et al. Ambulatory blood pressure after therapeutic and subtherapeutic nasal continuous positive airway pressure for obstructive sleep apnoea: a randomised parallel trial. Lancet 2002;359:204-10.

19. Mansfield DR, Gollogly NC, Kaye DM, et al. Controlled trial of continuous positive airway pressure in obstructive sleep apnea and heart failure. Am J Respir Crit Care Med 2004;169:361-6.

20. Kaneko Y, Floras JS, Usui K, et al. Cardiovascular effects of continuous positive airway pressure in patients with heart failure and obstructive sleep apnea. N Engl J Med 2003:348:1233-41.

21. Ross R. Atherosclerosis: an inflammatory disease. N Engl J Med 1999;340:115-26.

22. Libby P. Inflammation in atherosclerosis. Nature 2002;420:868-74.

23. Glass CK, Witztum JL. Atherosclerosis. the road ahead. Cell 2001;104:503-16.

24. Lusis AJ. Atherosclerosis. Nature 2000;407:233-41.

25. Pasceri V, Willerson JT, Yeh ET. Direct proinflammatory effect of C-reactive protein on human endothelial cells. Circulation 2000;102:2165-8.

26. Ciftci TU, Kokturk O, Bukan N, et al. The relationship between serum cytokine levels with obesity and obstructive sleep apnea syndrome. Cytokine 2004;28:87-91.

27. Dyugovskaya L, Lavie P, Lavie L. Phenotypic and functional characterization of blood gammadelta T cells in sleep apnea. Am J Respir Crit Care Med 2003;168:242-9.

28. Ryan S, Taylor CT, McNicholas WT. Selective activation of inflammatory pathways by intermittent hypoxia in obstructive sleep apnea syndrome. Circulation 2005; 112:2660-7.

29. Ryan S, Taylor CT, McNicholas WT. Predictors of elevated nuclear factor-kappaBdependent genes in obstructive sleep apnea syndrome. Am J Respir Crit Care Med 2006;174:824-30.

30. Minoguchi K, Tazaki T, Yokoe T, et al. Elevated production of tumor necrosis factoralpha by monocytes in patients with obstructive sleep apnea syndrome. Chest 2004;126:1473-9.

31. Gerszten RE, Garcia-Zepeda EA, Lim YC, et al. MCP-1 and IL-8 trigger firm adhesion of monocytes to vascular endothelium under flow conditions. Nature 1999;398:718-23.

32. Aukrust $\mathbf{P}$, Yndestad A, Smith C, et al. Chemokines in cardiovascular risk prediction. Thromb Haemost 2007;97:748-54.

33. Ohga $\mathbf{E}$, Tomita $\mathrm{T}$, Wada $\mathrm{H}$, et al. Effects of obstructive sleep apnea on circulating ICAM-1, IL-8, and MCP-1. J App/ Physiol 2003;94:179-84.

34. Yokoe T, Minoguchi $\mathrm{K}$, Matsuo $\mathrm{H}$, et al. Elevated levels of $\mathrm{C}$-reactive protein and interleukin- 6 in patients with obstructive sleep apnea syndrome are decreased by nasal continuous positive airway pressure. Circulation 2003;107:1129-34.

35. Vgontzas AN, Papanicolaou DA, Bixler EO, et al. Elevation of plasma cytokines in disorders of excessive daytime sleepiness: role of sleep disturbance and obesity. J Clin Endocrinol Metab 1997;82:1313-6.

36. Mehra R, Storfer-Isser A, Kirchner HL, et al. Soluble interleukin 6 receptor: a novel marker of moderate to severe sleep-related breathing disorder. Arch Intern Med 2006;166:1725-31.

37. Ueda K, Takahashi M, Ozawa K, et al. Decreased soluble interleukin-6 receptor in patients with acute myocardial infarction. Am Heart $J$ 1999;138:908-15.

38. Ohga E, Nagase T, Tomita T, et al. Increased levels of circulating ICAM-1, VCAM-1, and L-selectin in obstructive sleep apnea syndrome. J App/ Physiol 1999;87:10-4.

39. El-Solh AA, Mador MJ, Sikka P, et al. Adhesion molecules in patients with coronary artery disease and moderate-to-severe obstructive sleep apnea. Chest 2002;121:1541-7.

40. Ursavas A, Karadag M, Rodoplu E, et al. Circulating ICAM-1 and VCAM-1 levels in patients with obstructive sleep apnea syndrome. Respiration 2007;74:525-32.

41. Zamarron-Sanz C, Ricoy-Galbaldon J, Gude-Sampedro F, et al. Plasma levels of vascular endothelial markers in obstructive sleep apnea. Arch Med Res 2006;37:552-5.

42. Chin K, Nakamura T, Shimizu K, et al. Effects of nasal continuous positive airway pressure on soluble cell adhesion molecules in patients with obstructive sleep apnea syndrome. Am J Med 2000;109:562-7.

43. Ridker PM, Cushman M, Stampfer MJ, et al. Inflammation, aspirin, and the risk of cardiovascular disease in apparently healthy men. N Engl J Med 1997;336:973-9.

44. Koenig W, Sund M, Frohlich M, et al. C-Reactive protein, a sensitive marker of inflammation, predicts future risk of coronary heart disease in initially healthy middleaged men: results from the MONICA (Monitoring Trends and Determinants in Cardiovascular Disease) Augsburg Cohort Study, 1984 to 1992. Circulation 1999;99:237-42

45. Heeschen C, Hamm CW, Bruemmer J, et al. Predictive value of C-reactive protein and troponin $T$ in patients with unstable angina: a comparative analysis. CAPTURE Investigators. Chimeric c7E3 AntiPlatelet Therapy in Unstable angina REfractory to standard treatment trial. J Am Coll Cardiol 2000;35:1535-42.

46. Haverkate F, Thompson SG, Pyke SD, et al. Production of C-reactive protein and risk of coronary events in stable and unstable angina. European Concerted Action on Thrombosis and Disabilities Angina Pectoris Study Group. Lancet 1997;349:462-6. 
47. Miller M, Zhan M, Havas S. High attributable risk of elevated C-reactive protein level to conventional coronary heart disease risk factors: the Third National Health and Nutrition Examination Survey. Arch Intern Med 2005;165:2063-8.

48. Khera A, de Lemos JA, Peshock RM, et al. Relationship between C-reactive protein and subclinical atherosclerosis: the Dallas Heart Study. Circulation 2006;113:38-43.

49. Cao JJ, Arnold AM, Manolio TA, et al. Association of carotid artery intima-media thickness, plaques, and C-reactive protein with future cardiovascular disease and allcause mortality: the Cardiovascular Health Study. Circulation 2007;116:32-8.

50. Yao M, Tachibana N, Okura M, et al. The relationship between sleep-disordered breathing and high-sensitivity C-reactive protein in Japanese men. Sleep 2006;29:661-5.

51. Taheri S, Austin D, Lin L, et al. Correlates of serum C-reactive protein (CRP): no association with sleep duration or sleep disordered breathing. Sleep 2007;30:991-6.

52. Kokturk 0, Ciftci TU, Mollarecep E, et al. Elevated C-reactive protein levels and increased cardiovascular risk in patients with obstructive sleep apnea syndrome. Int Heart J 2005; $\mathbf{4 6}: 801-9$

53. Shamsuzzaman AS, Winnicki M, Lanfranchi $P$, et al. Elevated C-reactive protein in patients with obstructive sleep apnea. Circulation 2002;105:2462-4.

54. Can M, Acikgoz S, Mungan G, et al. Serum cardiovascular risk factors in obstructive sleep apnea. Chest 2006;129:233-7.

55. Barcelo A, Barbe F, Llompart E, et al. Effects of obesity on C-reactive protein level and metabolic disturbances in male patients with obstructive sleep apnea. Am J Med 2004;117:118-21.

56. Guilleminault C, Kirisoglu C, Ohayon MM. C-reactive protein and sleep-disordered breathing. Sleep 2004;27:1507-11.

57. Ryan S, Nolan GM, Hannigan E, et al. Cardiovascular risk markers in obstructive sleep apnoea syndrome and correlation with obesity. Thorax 2007:62:509-14.

58. Akashiba T, Akahoshi T, Kawahara S, et al. Effects of long-term nasal continuous positive airway pressure on C-reactive protein in patients with obstructive sleep apnea syndrome. Intern Med 2005:44:899-900.

59. Steiropoulos $\mathbf{P}$, Tsara V, Nena E, et al. Effect of continuous positive airway pressure treatment on serum cardiovascular risk factors in patients with obstructive sleep apnea-hypopnea syndrome. Chest 2007;132:843-51.

60. Kohler MM, Ayers L, Pepperell JCMF, et al. Effects of continuous positive airway pressure on systemic inflammation in patients with moderate to severe obstructive sleep apnoea: a randomised controlled trial. Thorax 2009;64:67-73.

61. Jelic S, Padeletti M, Kawut SM, et al. Inflammation, oxidative stress, and repair capacity of the vascular endothelium in obstructive sleep apnea. Circulation 2008; 117:2270-8.

62. Nacher M, Serrano-Mollar A, Farre R, et al. Recurrent obstructive apneas trigger early systemic inflammation in a rat model of sleep apnea. Respir Physiol Neurobiol 2007;155:93-6.

63. Dyugovskaya L, Lavie $\mathrm{P}$, Lavie L. Increased adhesion molecules expression and production of reactive oxygen species in leukocytes of sleep apnea patients. Am J Respir Crit Care Med 2002:165:934-9.

64. Lattimore JD, Wilcox I, Nakhla S, et al. Repetitive hypoxia increases lipid loading in human macrophages: a potentially atherogenic effect. Atherosclerosis 2005; 179:255-9.

65. Dyugovskaya L, Lavie P, Lavie L. Lymphocyte activation as a possible measure of atherosclerotic risk in patients with sleep apnea. Ann N Y Acad Sci 2005:1051:340-50.

66. Dyugovskaya L, Lavie $\mathrm{P}$, Hirsh $\mathrm{M}$, et al. Activated CD8+ T-lymphocytes in obstructive sleep apnoea. Eur Respir J 2005;25:820-8.

67. Dyugovskaya L, Polyakov A, Lavie P, et al. Delayed neutrophil apoptosis in sleep apnea patients. Am J Respir Crit Care Med 2008;177:544-54.

68. Brooks D, Horner RL, Kozar LF, et al. Obstructive sleep apnea as a cause of systemic hypertension. Evidence from a canine model. J Clin Invest 1997:99:106-9.

69. Fletcher EC, Lesske J, Qian W, et al. Repetitive, episodic hypoxia causes diurna elevation of blood pressure in rats. Hypertension 1992:19:555-61.

70. Fletcher EC, Lesske J, Culman J, et al. Sympathetic denervation blocks blood pressure elevation in episodic hypoxia. Hypertension 1992;20:612-9.
71. Bao G, Metreveli N, Li R, et al. Blood pressure response to chronic episodic hypoxia: role of the sympathetic nervous system. J Appl Physiol 1997:83:95-101.

72. Lai CJ, Yang CC, Hsu YY, et al. Enhanced sympathetic outflow and decreased baroreflex sensitivity are associated with intermittent hypoxia-induced systemic hypertension in conscious rats. J Appl Physiol 2006;100:1974-82.

73. Savransky V, Nanayakkara A, Li J, et al. Chronic intermittent hypoxia induces atherosclerosis. Am J Respir Crit Care Med 2007:175:1290-7.

74. Dematteis M, Julien C, Guillermet C, et al. Intermittent hypoxia induces early functional cardiovascular remodeling in mice. Am J Respir Crit Care Med 2008:177:227-35

75. Greenberg H, Ye X, Wilson D, et al. Chronic intermittent hypoxia activates nuclear factor-kappaB in cardiovascular tissues in vivo. Biochem Biophys Res Commun 2006;343:591-6.

76. Yamauchi M, Tamaki S, Tomoda K, et al. Evidence for activation of nuclear facto kappaB in obstructive sleep apnea. Sleep Breath 2006;10:189-93.

77. Ryan S, McNicholas WT, Taylor CT. A critical role for p38 map kinase in NF-kappaB signaling during intermittent hypoxia/reoxygenation. Biochem Biophys Res Commun 2007:355:728-33.

78. Kotlyarov A, Neininger A, Schubert C, et al. MAPKAP kinase 2 is essential for LPS induced TNF-alpha biosynthesis. Nat Cell Biol 1999;1:94-7.

79. Behr TM, Nerurkar SS, Nelson AH, et al. Hypertensive end-organ damage and premature mortality are p38 mitogen-activated protein kinase-dependent in a rat model of cardiac hypertrophy and dysfunction. Circulation 2001:104:1292-8.

80. Cain BS, Meldrum DR, Meng X, et al. p38 MAPK inhibition decreases TNF-alpha production and enhances postischemic human myocardial function. J Surg Res 1999;83:7-12.

81. Cook SA, Sugden PH, Clerk A. Activation of c-Jun N-terminal kinases and p38mitogen-activated protein kinases in human heart failure secondary to ischaemic heart disease. J Mol Cell Cardiol 1999:31:1429-34.

82. Li M, Georgakopoulos D, Lu G, et al. p38 MAP kinase mediates inflammatory cytokine induction in cardiomyocytes and extracellular matrix remodeling in heart. Circulation 2005:111:2494-502.

83. Liao P, Wang S0, Wang S, et al. p38 Mitogen-activated protein kinase mediates a negative inotropic effect in cardiac myocytes. Circ Res 2002;90:190-6.

84. Zarubin T, Han J. Activation and signaling of the p38 MAP kinase pathway. Cell Res 2005; 15:11-8.

85. Beguin PC, Belaidi E, Godin-Ribuot D, et al. Intermittent hypoxia-induced delayed cardioprotection is mediated by PKC and triggered by p38 MAP kinase and Erk1/2. J Mol Cell Cardiol 2007:42:343-51.

86. Yuan G, Nanduri J, Bhasker CR, et al. Ca2+/calmodulin kinase-dependent activation of hypoxia inducible factor 1 transcriptional activity in cells subjected to intermittent hypoxia. J Biol Chem 2005;280:4321-8.

87. Peng YJ, Yuan G, Ramakrishnan D, et al. Heterozygous HIF-1alpha deficiency impairs carotid body-mediated systemic responses and reactive oxygen species generation in mice exposed to intermittent hypoxia. J Physiol 2006;577:705-16.

88. Shaulian E, Karin M. AP-1 as a regulator of cell life and death. Nat Cell Biol 2002:4:E131-6.

89. Greenberg HE, Sica AL, Scharf SM, et al. Expression of c-fos in the rat brainstem after chronic intermittent hypoxia. Brain Res 1999;816:638-45.

90. Goldbart A, Row BW, Kheirandish L, et al. Intermittent hypoxic exposure during light phase induces changes in CAMP response element binding protein activity in the rat CA1 hippocampal region: water maze performance correlates. Neuroscience 2003;122:585-90.

91. Lavie L. Obstructive sleep apnoea syndrome: an oxidative stress disorder. Sleep Med Rev 2003; 7:35-51.

92. Hayakawa $\mathbf{M}$, Miyashita $\mathrm{H}$, Sakamoto I, et al. Evidence that reactive oxygen species do not mediate NF-kappaB activation. Embo J 2003;22:3356-66.

93. Alam I, Lewis K, Stephens JW, et al. Obesity, metabolic syndrome and sleep apnoea: all pro-inflammatory states. Obes Rev 2007:8:119-27.

94. Fantuzzi G. Adipose tissue, adipokines, and inflammation. J Allergy Clin Immunol 2005;115:911-20. 